\title{
Histone variants: dynamic punctuation in transcription
}

\author{
Christopher M. Weber ${ }^{1,2}$ and Steven Henikoff ${ }^{1,3,4}$ \\ ${ }^{1}$ Division of Basic Sciences, Fred Hutchinson Cancer Research Center, Seattle, Washington 98109, USA; ${ }^{2}$ Molecular and Cellular \\ Biology Program, University of Washington, Seattle, Washington 98195, USA; ${ }^{3}$ Howard Hughes Medical Institute, Fred \\ Hutchinson Cancer Research Center, Seattle, Washington 98109, USA
}

Eukaryotic gene regulation involves a balance between packaging of the genome into nucleosomes and enabling access to regulatory proteins and RNA polymerase. Nucleosomes are integral components of gene regulation that restrict access to both regulatory sequences and the underlying template. Whereas canonical histones package the newly replicated genome, they can be replaced with histone variants that alter nucleosome structure, stability, dynamics, and, ultimately, DNA accessibility. Here we consider how histone variants and their interacting partners are involved in transcriptional regulation through the creation of unique chromatin states.

The basic unit of chromatin is the nucleosome, consisting of DNA wrapped around a core of histone proteins. Nucleosomes likely evolved to protect and compact increasingly large eukaryotic genomes (Malik and Henikoff 2003). As a consequence, however, nucleosomes also restrict access to cellular components such as DNAbinding transcription factors and RNA polymerase ( $\mathrm{Li}$ et al. 2007). Accordingly, distinct mechanisms have evolved to influence the dynamic competition between nucleosomes and DNA-binding transcription factors in addition to orchestrating RNA polymerase II (RNAPII) translocation across a nucleosomal template. This dynamic mode of regulation is mediated in a number of ways, including post-translational modification of histones, altering the position or eviction of nucleosomes by ATP-dependent chromatin remodelers, and replacement of canonical histones with histone variants. Canonical histones (H2A, H2B, H3, and $\mathrm{H} 4)$ are deposited in a replication-coupled manner to package the newly replicated genome. In contrast, histone variants are expressed throughout the cell cycle and replace canonical histones or take their place when nucleosomes are evicted (for reviews on evolutionary conservation, see Malik and Henikoff 2003; Talbert and Henikoff 2010). Histone variants have dis-

[Keywords: gene regulation; histone chaperones; nucleosome dynamics; nucleosome remodeling]

${ }^{4}$ Corresponding author

E-mail steveh@fhcrc.org

Article is online at http://www.genesdev.org/cgi/doi/10.1101/gad.238873.114. Freely available online through the Genes \& Development Open Access option. tinct amino acid sequences that can influence both the physical properties of the nucleosome and nucleosome dynamics. These properties are especially important during transcription, where histone variants shape the chromatin landscape of cis-regulatory and coding regions in support of specific transcription programs.

Here we consider core variants of $\mathrm{H} 2 \mathrm{~A}$ and $\mathrm{H} 3$ that are implicated in transcription (Table 1). We discuss the mechanisms responsible for shaping the histone variant landscape, focusing on recent genome-wide mapping studies of these variants, their chaperones, RNAPII, and nucleosome dynamics. We review how these chromatin landscapes and deposition pathways influence the dynamic interplay between nucleosome occupancy, regulatory DNA-binding proteins, and, ultimately, RNAPII elongation across nucleosomes. Our ultimate focus is on how histone variants create distinct chromatin landscapes with different dynamics and how this influences gene regulation.

\section{Nucleosome organization and dynamics}

Each nucleosome wraps $\sim 147$ base pairs (bp) of DNA 1.7 turns around an octamer consisting of two each of $\mathrm{H} 2 \mathrm{~A}$, $\mathrm{H} 2 \mathrm{~B}, \mathrm{H} 3$, and H4. At the center of the DNA wrap, an $(\mathrm{H} 3 /$ $\mathrm{H} 4)_{2}$ tetramer is formed due to a strong four-helix bundle interaction between the two $\mathrm{H} 3$ proteins (Luger et al. 1997). Interacting with the $(\mathrm{H} 3 / \mathrm{H} 4)_{2}$ tetramer are two heterodimers of $\mathrm{H} 2 \mathrm{~A} / \mathrm{H} 2 \mathrm{~B}$, which dock at the DNA entry and exit sites through the $\mathrm{H} 2 \mathrm{~A} \mathrm{C}$ terminus-docking domain (Fig. 1A). Additionally, the two H2A histones interact through their L1 loop, and H2B interacts with $\mathrm{H} 4$ through a weak four-helix bundle.

Nucleosomes are energetically stable; however, they can turn over in vivo (Fig. 1B). H2A and H2B turn over much faster than $\mathrm{H} 3$ and $\mathrm{H} 4$ in both genic and intergenic regions (Kimura and Cook 2001; Jamai et al. 2007). H2A/ $\mathrm{H} 2 \mathrm{~B}$ turnover occurs due to weaker intranucleosomal contacts and because the dimers dock at DNA entry and exit sites, which are prone to transiently unwrap (Li et al. 2005b). Active processes such as RNA polymerase transit

(C) 2014 Weber and Henikoff This article, published in Genes \& Development, is available under a Creative Commons License (Attribution 4.0 International), as described at http://creativecommons.org/licenses/by/4.0. 
Table 1. Histone variants discussed in this review and the lineages where they are present

\begin{tabular}{ll}
\hline Histone variant & \multicolumn{1}{c}{ Presence } \\
\hline H2A.Z & Universal \\
macroH2A & \\
macroH2A & Invertebrates \\
macroH2A.1.1/2 & Vertebrates \\
macroH2A.2 & Vertebrates \\
H2A.B & Vertebrates \\
H2A.Lap1 & Mouse \\
H2A.Bbd & Human \\
H3.3 & Universal (only H3 in fungi) \\
\hline
\end{tabular}

drive unwrapping, which increases dimer turnover and DNA exposure (Sheinin et al. 2013). A functional consequence of both transient unwrapping and dimer loss is exposure of DNA to binding by regulatory proteins.

In order for the $(\mathrm{H} 3 / \mathrm{H} 4)_{2}$ tetramer to turn over, the nucleosome must be almost completely unwrapped, a process that can occur many times at a given location during interphase. In yeast and Drosophila cells, nucleosome turnover measured by new $\mathrm{H} 3$ incorporation correlates with transcription (Dion et al. 2007; Deal et al. 2010). In Drosophila cells, nucleosome turnover is also high over cis-regulatory regions, consistent with a competition between nucleosomes and transcription factors for occupancy of these sites (Mito et al. 2007; Deal et al. 2010). A consequence of the dynamic nature of nucleosomes is erasure of post-translational modifications on histones. Thus, modulation of DNA accessibility through regulated nucleosome turnover can perpetuate gene expression states.

\section{Replication-independent deposition of variants punctuates the chromatin landscape}

\section{H2A.Z alters nucleosome properties}

$\mathrm{H} 2 \mathrm{~A}$ variants are the most diverse, perhaps reflective of relaxed structural constraint within the nucleosome. One such variant, H2A.Z, arose once early in eukaryotic evolution and has remained distinct from $\mathrm{H} 2 \mathrm{~A}$ ever since (Talbert and Henikoff 2010). At the amino acid level, $\mathrm{H} 2 \mathrm{~A}$. $\mathrm{Z}$ is only $\sim 60 \%$ identical to $\mathrm{H} 2 \mathrm{~A}$ within species but is relatively conserved between species and is essential in metazoans (Zlatanova and Thakar 2008). Remarkably, the structure of the H2A.Z nucleosome is quite similar to $\mathrm{H} 2 \mathrm{~A}$; however, there are key structural differences (Suto et al. 2000). On the surface, H2A.Z has an extended acidic patch, which stimulates remodeling activity with the ISWI ATP-dependent remodeler (Goldman et al. 2010). Within the core, the L1 loop is structurally distinct, and in the docking domain with $\mathrm{H} 3 / \mathrm{H} 4$, a glutamine-toglycine substitution in H2A.Z compromises three hydrogen bonds, which is predicted to weaken the interaction. Despite these structural differences, the change in the stability of the particle is subtle, with contrasting results reported. Overall, the consensus is that H2A.Z slightly stabilizes in vitro and destabilizes in vivo (Zlatanova and Thakar 2008; Bonisch and Hake 2012). This discrepancy might be attributable to post-translational modifications in vivo, where $\mathrm{H} 2 \mathrm{~A} . \mathrm{Z}$ is acetylated at active genes (Bruce et al. 2005; Valdes-Mora et al. 2012); differences in DNA sequence; or the fact that H2A.Z nucleosomes can be hybrid in vivo, either heterotypic (Z/A) or homotypic (Z/Z) (Viens et al. 2006; Luk et al. 2010; Weber et al. 2010).

\section{H2A.Z deposition and a futile cycle}

In yeast, H2A.Z can be bound by the general H2A/H2B chaperone Nap1 or Chz1, which preferentially bind H2A.Z over H2A (Luk et al. 2007). These chaperones provide a source of H2A.Z for the Swr1 remodeling complex, which exchanges H2A.Z for H2A (Mizuguchi et al. 2004). Some metazoans contain two Swr1 orthologs that organize into at least two distinct complexes, P400/TIP60 and SRCAP, which, like Swr1, catalyze the exchange reaction. While these complexes share some components, there are many differences (Billon and Cote 2012). For
A

B
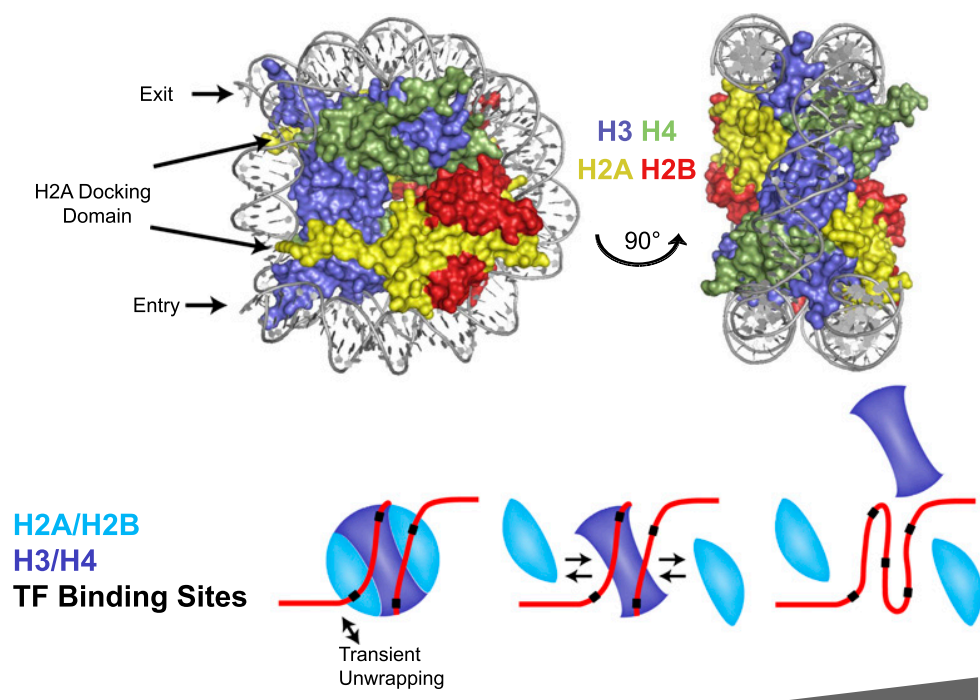

Accessibility
Figure 1. (A) Structure of nucleosome core particle, showing that $(\mathrm{H} 3 / \mathrm{H} 4)_{2}$ is at the center of the DNA wrap, with two dimers of $\mathrm{H} 2 \mathrm{~A} / \mathrm{H} 2 \mathrm{~B}$ docked at the edges, near the DNA entry and exit locations. (B) Nucleosomes modulate access to transcription factor (TF)-binding sites. Transient DNA unwrapping exposes transcription factorbinding sites. As nucleosomes unwrap, $\mathrm{H} 2 \mathrm{~A} / \mathrm{H} 2 \mathrm{~B}$ dimers can be lost, exposing more DNA, and when the nucleosome is completely unwrapped, $(\mathrm{H} 3 / \mathrm{H} 4)_{2}$ is lost, and DNA is completely exposed. 
example, a human H2A.Z-specific chaperone, ANP32E, was recently characterized as part of P400/TIP60 but not the SRCAP complex (Obri et al. 2014). Intriguingly, the ANP32 family has many members of uncharacterized function, where at least one other, ANP32B, has been shown to be an $\mathrm{H} 3 / \mathrm{H} 4$ chaperone (Tochio et al. 2010). It is likely that many other histone chaperones remain to be discovered.

H2A.Z comprises $\sim 15 \%$ of total H2A and is distributed throughout the genome nonrandomly in both euchromatin and heterochromatin, where it is monoubiquitinated (Sarcinella et al. 2007); however, it has remained unclear how H2A.Z becomes enriched at particular sites in the genome. High-resolution ChIP (chromatin immunoprecipitation) and biochemistry of the yeast Swrl complex revealed that Swrl preferentially acts at nucleosomedepleted regions (NDRs) that are $>50-70$ bp and requires the Swc2 subunit to bind DNA (Ranjan et al. 2013; Yen et al. 2013). NDRs are predominately located at the promoter region of active genes and are characterized by two well-positioned flanking nucleosomes. Certain transcription factors and chromatin remodelers are required for NDR establishment and maintenance, but H2A.Z and Swr1 are dispensable (Whitehouse et al. 2007; Hartley and Madhani 2009). Whereas recruitment to the NDR might be sufficient to explain H2A.Z enrichment at nucleosomes that flank promoters, H2A.Z is also enriched to some extent in gene bodies of all eukaryotes studied. Additionally, NDR recruitment does not explain how some organisms, including Arabidopsis (Zilberman et al. 2008) and Drosophila (Mavrich et al. 2008), lack upstream H2A.Z nucleosomes (Fig. 2A).

In metazoans, H2A.Z enrichment correlates with expression level. One attractive possibility is that dimer loss followed by replacement with H2A.Z contributes to genic enrichment patterns and low-level incorporation genome-wide. In support of this possibility, homotypic $\mathrm{Z} / \mathrm{Z}$ nucleosomes are enriched over active genes (Weber et al. 2010; Nekrasov et al. 2012), which could be the result of transcription-mediated dimer loss and then opportunistic replacement with H2A.Z, which is produced throughout the cell cycle. Consistent with transcriptioncoupled replacement, the presence of upstream H2A.Z nucleosomes seen in some organisms correlates with bidirectional transcription in yeast and mammals (Core et al. 2008; Xu et al. 2009), whereas upstream H2A.Z enrichment is not seen in Arabidopsis and Drosophila. DNA methylation and H2A.Z are anti-correlated in plants and animals (Conerly et al. 2010; Zemach et al. 2010), and in Arabidopsis, there is mutual antagonism (Zilberman et al. 2008), suggesting that epigenetic factors also contribute to H2A.Z localization.

In mammalian cells, preferential H2A.Z localization also is seen at a subset of gene promoters associated with transcription factor binding (Gevry et al. 2007; GallantBehm et al. 2012). Additionally, work in embryonic stem cells (ESCs) has shown that H2A.Z preferentially localizes to promoters of silent genes occupied by the Polycomb-repressive complex 2 (PRC2) (Creyghton et al. 2008; Illingworth et al. 2012). H2A.Z is also enriched at

\section{A H2A.Z Futile Cycle}

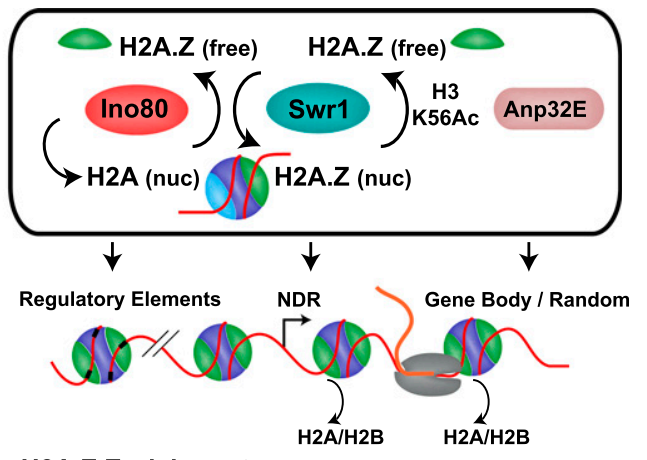

H2A.Z Enrichment

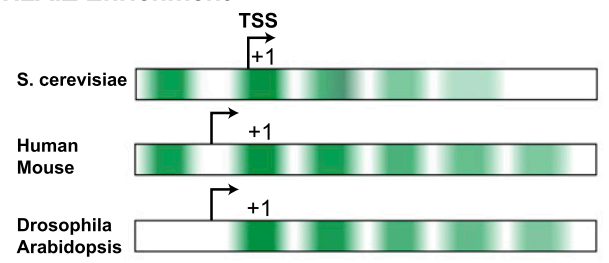

B macroH2A Deposition

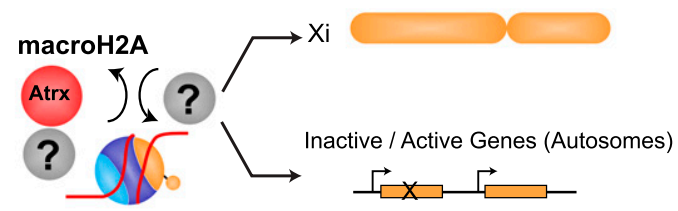

C H3.3 Deposition

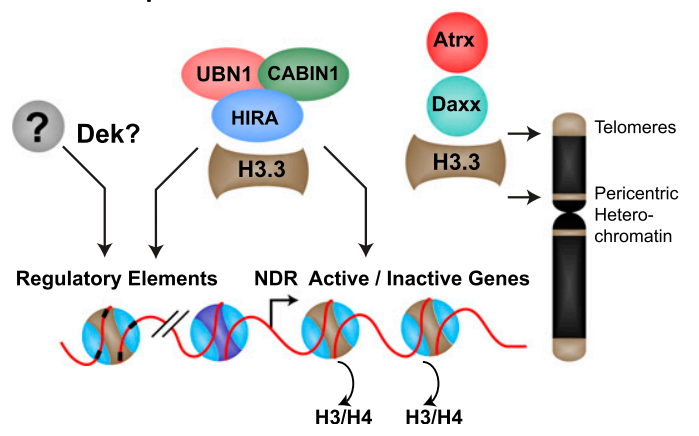

Figure 2. $(A, t o p)$ The H2A.Z futile cycle of deposition by Swr1 or orthologous complexes and then removal by Ino80, Anp32E, or Swr1 when H3K56 is acetylated. (Bottom) H2A.Z enrichment patterns and promoter architecture differences in different organisms. $(B)$ macroH2 $\mathrm{A}$ is deposited in the inactive $\mathrm{X}(\mathrm{Xi})$ and inactive/active genes on autosomes. (C) H3.3 deposition is mediated by the HIRA complex at genes and regulatory elements, whereas Atrx and Daxx mediate H3.3 incorporation at telomeres and pericentric heterochromatin. It is not clear how H3.3 is enriched at some cis-regulatory elements.

enhancers in ESCs and facilitates the binding of PRC2 and H3K4me3 and H3K27me3 modifications (Hu et al. 2013). Also in ESCs, H2A.Z increases nucleosome accessibility at FoxA2-binding sites (Li et al. 2012) and transcription factor accessibility (Hu et al. 2013). Despite these intriguing observations, it remains unclear how H2A.Z becomes enriched at developmentally regulated regions. 
H2AZ can be actively removed from chromatin. For example, the recently described ANP32E chaperone specifically removes H2A.Z from nucleosomes, including those at cis-regulatory sites (Obri et al. 2014). The Ino80 complex catalyzes replacement of $\mathrm{H} 2 \mathrm{~A}$.Z with canonical H2A (Papamichos-Chronakis et al. 2011), just the reverse of the Swr1 complex, thus completing a futile cycle when both complexes act successively on the same nucleosome. In an Ino80 mutant, H2A.Z is globally mislocalized, suggesting that Ino80 clears out H2A.Z that is spuriously incorporated. Ino80 is also found at yeast NDRs, where high-resolution ChIP suggests that Nhp10, les5, and Arp8 subunits are important for binding, similar to the Swc2 subunit of the Swr1 complex (Yen et al. 2013). In addition, Swr1 can carry out the reverse reaction when H3K56 is acetylated in yeast (Watanabe et al. 2013). However, when Swc2 is present, this activity is inhibited, suggesting that this subunit functions as a lock to prevent further remodeling. In yeast, H3K56 is acetylated during replication-coupled nucleosome assembly and is present on $\sim 30 \%$ of total histone $\mathrm{H} 3$ (Xu et al. 2005), whereas it marks $<1 \%$ in human cells (Xie et al. 2009). Considering its paucity in metazoans, it remains unclear how general a role H3K56ac plays in removal of H2A.Z.

\section{Other H2A variants: life on the edge}

Unlike H2A.Z, which is nearly universal across eukaryotes, other replication-independent $\mathrm{H} 2 \mathrm{~A}$ variants implicated in transcription have evolved only in animals. For example, H2A.B, first described as H2A.Bbd (Barr bodydeficient) has been found only in mammals. H2A.B is only $\sim 50 \%$ identical to $\mathrm{H} 2 \mathrm{~A}$ and is rapidly evolving (Ishibashi et al. 2010). The C terminus of H2A.B is 19 amino acids shorter than that of $\mathrm{H} 2 \mathrm{~A}$, reducing the tail and part of the docking domain. H2A.B lacks an acidic patch on its surface and is also referred to as H2A.Lap1 (lacks acidic patch 1) (Soboleva et al. 2012). These modifications to the structure substantially alter the physical properties of H2A.B-containing nucleosomes. For example, human H2A.B nucleosome arrays inhibit the formation of compact chromatin fibers (Zhou et al. 2007). Mouse H2A.B can form partially compacted arrays due to a single aspartate residue in the acidic patch, which is not found in humans (Soboleva et al. 2012). Consistent with an integral role for the docking domain in modulating nucleosome stability, H2A.B nucleosomes are less stable, protect $\sim 30$ bp less DNA against micrococcal nuclease digestion, and exchange much faster by FRAP than canonical H2A (Bao et al. 2004; Gautier et al. 2004; Doyen et al. 2006b; Bonisch and Hake 2012; Tolstorukov et al. 2012). These effects are largely attributable to the docking domain because adding the H2A C-terminal tail, including the docking domain, onto H2A.B partially reverses the instability (Doyen et al. 2006b).

Currently, it remains unclear whether there are specific chaperones or mechanisms for H2A.B deposition. However, the general chaperone NAP-1 can efficiently assemble and disassemble H2A.B dimers in vitro /Okuwaki et al. 2005). In HeLa cells, ectopically expressed H2A.B localizes over gene bodies and correlates with expression level (Tolstorukov et al. 2012). Similarly, in ESCs, endogenous H2A.B is enriched over the body of actively transcribed genes (Chen et al. 2014). In contrast, in mouse testis where H2A.B is especially abundant, it is enriched over gene promoters and lowly over gene bodies (Soboleva et al. 2012). It remains unclear why H2A.B localization is different; nonetheless, H2A.B deposition results in a distinct chromatin landscape that is destabilized and less compact.

Another $\mathrm{H} 2 \mathrm{~A}$ variant implicated in transcription, macroH2A, is distinct from all other histones in that it contains a nonhistone globular (macro) domain. The macro domain on the $\mathrm{C}$ terminus is connected through an unstructured linker to a histone fold domain that is $\sim 60 \%$ identical to canonical $\mathrm{H} 2 \mathrm{~A}$, resulting in a histone protein that is approximately three times the size of H2A (Chakravarthy et al. 2005). Macro domain-containing proteins are found in many organisms; however, macroH2A is restricted to vertebrates and a few invertebrates (Talbert and Henikoff 2010). Macro domains are known to bind metabolites of $\mathrm{NAD}^{+}$, including poly(ADP-ribose), and have distinct biological roles, including transcriptional regulation (Han et al. 2011). In vertebrates, there are three macroH2A isoforms: macroH2A.1.1, macroH2A.1.2, and macroH2A.2. The first two are splice isoforms from a single gene, whereas a separate gene encodes the latter. However, only macroH2A.1.1 is capable of binding metabolites of $\mathrm{NAD}^{+}$. In contrast to both H2A.Z and H2A.B, macroH2A preferentially forms heterotypic nucleosomes in vitro, whereas H2A.Z shows no preference (Chakravarthy et al. 2004; Chakravarthy and Luger 2006). macroH2A has a higher salt-dependent stability than $\mathrm{H} 2 \mathrm{~A}$, where four residue changes in the L1 loop are most important (Abbott et al. 2005; Chakravarthy and Luger 2006).

In vivo, macroH2A is enriched on the transcriptionally inactivated female $\mathrm{X}$ chromosome, senescence-associated heterochromatic foci (SAHF), and large transcriptionally silent domains (Costanzi and Pehrson 1998; Zhang et al. 2005; Gamble et al. 2010; Tolstorukov et al. 2012). macroH2A localization to the inactive $\mathrm{X}$ is disrupted when Xist RNA is deleted, suggesting a role for this essential cisregulatory RNA in macroH2A recruitment (Csankovszki et al. 1999). Both the histone and nonhistone segments of macroH2A are sufficient for targeting to the inactive $\mathrm{X}$ (Chadwick et al. 2001; Nusinow et al. 2007). In SAHF, macroH2A deposition is promoted by histone regulator A (HIRA), a chaperone responsible for histone variant $\mathrm{H} 3.3$ incorporation at genes, and the nucleosome assembly and disassembly factor Asf1 (Zhang et al. 2005). Rather than directly deposit macroH2A, these chaperones might help to clear the way for macroH2A deposition. Consistent with higher stability and a generally repressive role, macroH2A appears to impair transcription factor binding and has been suggested to impair remodeling by SWI/SNF and ISWI (Angelov et al. 2003). Although more recent results suggest that macroH $2 \mathrm{~A}$ is an excellent substrate for remodeling by these remodelers, macroH $2 \mathrm{~A}$ reduces recruitment of the SWI/SNF remodeler (Chang et al. 2008). macroH2A also 
associates with the SWI/SNF family DNA translocase ATRX ( $\alpha$-thalassemia/MR, X-linked), which, like Ino80 for H2A.Z, negatively regulates macroH2A deposition (Fig. 2B; Ratnakumar et al. 2012). Despite identification of these partners for macroH2A, it remains unclear how it is localized into specific genomic regions.

\section{H3.3: filling gaps and more}

When a nucleosome is lost independent of replication, the $(\mathrm{H} 3 / \mathrm{H} 4)_{2}$ tetramer is replaced by the $\mathrm{H} 3.3$ variant and its $\mathrm{H} 4$ partner. Most eukaryotes express canonical $\mathrm{H} 3$ for replication-coupled deposition and the replication-independent H3.3 variant; however, some eukaryotes, such as fungi, express only the H3.3 type (Malik and Henikoff 2003). In metazoans, H3.3 differs from H3 by only four to five amino acids (Filipescu et al. 2013). Three of these differences are found within the core histone fold domain and specify the alternative deposition pathways (Ahmad and Henikoff 2002). The fraction of the genome occupied by H3.3 is variable, depending largely on dilution by canonical H3 during replication. For example, H3.3 comprises $\sim 90 \%$ of the histone 3 in terminally differentiated neurons (Pina and Suau 1987); however, in dividing cells, H3.3 comprises only 20\% (McKittrick et al. 2004). H3.3 incorporation is largely opportunistic, occurring when DNA is exposed at dynamic regions such as gene promoters, the body of active genes, and cis-regulatory elements (Mito et al. 2007; Ray-Gallet et al. 2011; Schneiderman et al. 2012). H3.3 was also found to be enriched over a subset of repressed genes in mammalian ESCs that exhibit lower dynamics, suggesting an expanded role for H3.3 beyond simply filling gaps in active chromatin (Goldberg et al. 2010). H3.3 enrichment over genes, both active and inactive, depends on the HIRA complex (Tagami et al. 2004). In addition, HIRA-independent mechanisms of H3.3 incorporation have been described (Banaszynski et al. 2013). For example, Daxx has been identified as a novel H3.3-specific chaperone, which, together with the SWI/SNF family remodeler Atrx, is responsible for incorporation at telomeres and pericentric heterochromatin (Drane et al. 2010; Goldberg et al. 2010). A single methionine-to-glycine substitution at position 90 in H3.3 appears to be a dominant contributor to the specificity of H3.3 interaction with the Daxx chaperone (Elsasser et al. 2012). In Drosophila, Daxx and Dek have been shown to deposit H3.3 in regulatory elements (Sawatsubashi et al. 2010).

From a structural perspective, H3 occupies the center of the nucleosome, and so it might not be surprising that the sequence of an $\mathrm{H} 3$ variant would be more constrained than is seen for variants of H2A. However, this constraint is not observed in the centromere-specific $\mathrm{H} 3$ variant cenH3, which shares only $\sim 50 \%-60 \%$ identity with $\mathrm{H} 3$ within the histone fold domain (Malik and Henikoff 2003). Considering that the alterations to H3.3 are subtle, it is not surprising that no destabilization attributable to H3.3 nucleosomes has been detected in vitro (Thakar et al. 2009; Chen et al. 2013). However, analysis of chromatin from chicken cells found that H3.3-containing nu- cleosomes are more sensitive to salt-dependent disruption and that H3.3/H2A.Z double-variant nucleosomes were most unstable (Jin and Felsenfeld 2007). This effect was independent of acetylation, a modification associated with destabilization of nucleosomes, suggesting that the effect is intrinsic or is due to incorporation at active regions of the genome. In support of the latter explanation, H3.3/H2A.Z nucleosomes are enriched over regulatory elements and NDRs, which are frequently disrupted (Jin et al. 2009). However, nucleosome turnover and HIRA binding to chromatin are reduced after H3.3 depletion (Banaszynski et al. 2013). HIRA was recently shown to directly interact with transcription factors and the Brgl chromatin remodeling complex (Pchelintsev et al. 2013). These results overall suggest that incorporation of $\mathrm{H} 3.3$ promotes a hyperdynamic state through its interaction partners within the nucleus.

\section{Histone variants in transcriptional regulation}

\section{H2A.Z: the positive, the negative, and the unknown}

A role for H2A.Z in transcription was initially proposed $>30$ years ago with the observation that Tetrahymena H2A.Z is present in the transcriptionally active macronucleus but not in the inactive micronucleus (Allis et al. 1980). Initial studies investigating the effect of H2A.Z on transcription were conducted in yeast, where it was shown to antagonize telomeric silencing, interact with activators, and help recruit RNAPII (Santisteban et al. 2000; Adam et al. 2001; Meneghini et al. 2003). Upon activation, H2A.Z is lost from 5' ends of genes, suggesting that it poises genes for activation by enabling access to the promoter/transcription start site (TSS). In human cells, H2A.Z is exchanged prior to RNAPII loading, likely as a consequence of promoter remodeling, where it has a role in RNAPII recruitment (Hardy et al. 2009). It is possible that the difference in the action of H2A.Z between yeast and metazoans stems from the fact that, in yeast, there is a nucleosome over the TSS that must be removed for RNAPII to load (Fig. 2A; Rhee and Pugh 2012). Regardless of the nucleosome architecture at promoters, H2A.Z consistently plays an activating role (Fig. 3).

In addition to roles in activation and initiation, H2A.Z has been found to promote elongation (Santisteban et al. 2011). To explore the molecular basis for this role, we developed a single-base-resolution method to map the position of RNAPII through the 3' end of nascent transcripts ( $\left.3^{\prime} \mathrm{NT}\right)$ in Drosophila. We found an anti-correlation between H2A.Z occupancy and RNAPII stalling as it transcribes across nucleosomes genome-wide (Weber et al. 2014). When H2A.Z levels were reduced both directly and by impairing Swrl activity, the nucleosome barrier to RNAPII increased. Our results favor a model in which $\mathrm{H} 2 \mathrm{~A} . \mathrm{Z} / \mathrm{H} 2 \mathrm{~B}$ dimers are more easily lost when the nucleosome is unwrapped, aiding RNAPII transcriptional elongation. It is also possible that elongation factors such as the FACT complex have increased activity with H2A.Z nucleosomes to ease the barrier or that acetylation "unlocks" H2A.Z nucleosomes by destabilizing them. For 


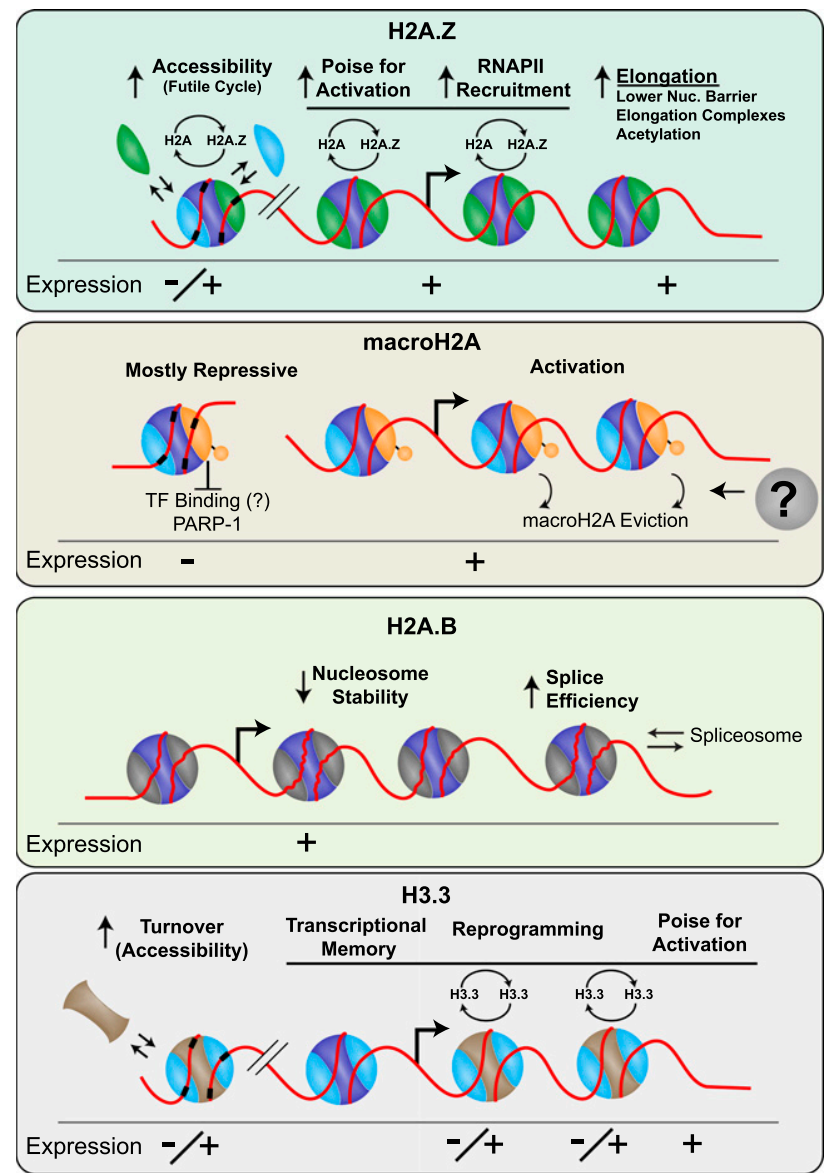

Figure 3. Models to explain the general role of histone variants and their deposition pathways on transcriptional regulation. Plus or minus expression level denotes the effect of the variant on transcriptional output.

example, in yeast, H2A.Z and Spt16, a component of the FACT complex, are synthetic-lethal (Biswas et al. 2006). Also, H2A.Z is hyperacetylated only when genes are expressed (Bruce et al. 2005; Valdes-Mora et al. 2012), and acetylation is required to achieve proper transcript levels (Halley et al. 2010; Valdes-Mora et al. 2012).

Despite a generally positive role for H2A.Z in transcription, negative effects have also been reported. For example, yeast h2a.zs mutants derepress silencing at the HMR locus (Dhillon and Kamakaka 2000), and whole-genome transcriptome analysis identified both positive and negative changes (Meneghini et al. 2003). In mammals, H2A.Z negatively regulates p21 and targets of the p63 transcription factor and occupies the promoters of genes that are silenced during mitosis (Gevry et al. 2007; Kelly et al. 2010; Gallant-Behm et al. 2012). Intriguingly, the h2a.zs phenotype, which extends beyond transcription defects, is partially suppressed by also mutating integral components of the Swr1 complex (Halley et al. 2010; Morillo-Huesca et al. 2010). This suggests that Swr1 activity in the absence of H2A.Z causes "mischief" by removing H2A but is unable to replace it. In the absence of both H2A.Z and Swr1, more yeast genes are down-regulated than upregulated (Morillo-Huesca et al. 2010). However, in
Arabidopsis, h2a.zs swr1s (pie1-5) mutants are worse off than either individual mutant, suggesting nonredundant functions for Swr1 (Coleman-Derr and Zilberman 2012). In Arabidopsis mutants that cannot incorporate H2A.Z, there is also global misregulation of transcription, with many genes up-regulated and many down-regulated. Interestingly, in Arabidopsis, H2A.Z is lost at elevated temperatures independent of transcription, and the transcriptome of the incorporation mutant resembles that of temperature-shifted plants (Kumar and Wigge 2010). Thus, in both yeast and plants, where H2A.Z mutants are viable, loss of the H2A.Z deposition pathway results in global effects on transcription.

How might H2A.Z function to exert both positive and negative effects on transcription? An attractive explanation is that H2A.Z facilitates binding of both activating and repressive complexes by keeping regions of the genome accessible. Support for this model comes from work in ESCs and during differentiation that has shown that H2A.Z facilitates binding of PRC2, MLL, and transcription factors (Creyghton et al. 2008; Li et al. 2012; $\mathrm{Hu}$ et al. 2013). In this context, H2A.Z deposition increases the fraction of the genome that is nucleasehypersensitive and decreases nucleosome occupancy at enhancers ( $\mathrm{Hu}$ et al. 2013). Interestingly, nucleosome depletion at transcription factor-binding sites is dependent on SWI/SNF and Ino80 chromatin remodeling complexes (Li et al. 2012). This suggests that a function of the futile cycle of deposition and removal might be to modulate accessibility to various regulatory proteins. It was previously suggested that H2A.Z regulates nucleosome positioning around promoters, which could influence accessibility of cis-regulatory regions (Guillemette et al. 2005; Marques et al. 2010). However, we and others failed to detect changes in nucleosome positioning following H2A.Z knockdown or deletion (Li et al. 2005a; Hartley and Madhani 2009; Weber et al. 2014). Although there might be intrinsic effects of $\mathrm{H} 2 \mathrm{~A} . \mathrm{Z}$ on translational or rotational positioning in yeast (Albert et al. 2007), it is also possible that these attributes are due to enriching for promoter-flanking nucleosomes, having little to do with the properties of H2A.Z.

Overall, H2A.Z functions to support transcriptional activation and elongation, which helps to explain its enrichment near promoters and over the coding region of most genes in eukaryotes. Future work will determine which components are involved in H2A.Z dynamics and how these accessible regions are regulated both positively and negatively. This is especially relevant in mammalian cells, which deposit H2A.Z through P400 and SRCAP complexes, whose independent functions are not yet clear. That H2A.Z functions to facilitate access to both repressive and active regulatory complexes explains the vexing dual nature of $\mathrm{H} 2 \mathrm{~A} . \mathrm{Z}$ in transcription and helps to explain why H2A.Z is essential in development.

\section{H2A.B}

Whereas the role of H2A.B in transcription has not been as thoroughly investigated as that of H2A.Z, recent ex- 
periments have provided interesting results. H2A.B knockdown in HeLa cells resulted in substantial changes in gene expression, with more genes down-regulated than up-regulated (Tolstorukov et al. 2012). In mouse ESCs, H2A.B knockdown resulted in a more modest effect on gene expression; however, most genes were down-regulated. Included in the differentially expressed set are a few imprinted genes where it was shown that H2A.B facilitates elongation over the differentially methylated region of the gene (Chen et al. 2014). Consistent with a role for H2A.B in transcriptional elongation, H2A.B has also been shown to associate with components of the spliceosome, and, upon knockdown, RNA splicing is less efficient (Tolstorukov et al. 2012). In mouse testis, H2A.B is associated with promoter regions, as discussed above, although it is not yet known whether it has any specific effect on expression (Soboleva et al. 2012). Overall, H2A.B appears to promote transcriptional elongation, likely as a consequence of creating less-stable nucleosomes that are more easily disrupted upon interaction with RNAPII (Fig. 3).

\section{macroH2A}

macroH2A localization on the inactivated female $\mathrm{X}$ chromosome, silent SAHF, and large transcriptionally silent domains (Costanzi and Pehrson 1998; Zhang et al. 2005; Gamble et al. 2010; Tolstorukov et al. 2012) suggests a role in transcriptional repression, although macroH2A is not required for X-chromosome inactivation (Changolkar et al. 2007). Genome-wide studies in human NT2 cells have shown that macroH2A is enriched at developmentally regulated genes, including overlap with the PRC2 complex. Nevertheless, it also represses transcription in vitro (Doyen et al. 2006a); in vivo, it represses IL8 transcription in a human B-cell line and endogenous retroviruses in mice (Agelopoulos and Thanos 2006; Changolkar et al. 2008). Knockdown of macroH2A increased the sensitivity of genes in the HOXA cluster to retinoic acid, further suggesting that it acts to repress transcription (Buschbeck et al. 2009). In the human breast cancer cell line MCF-7, most genes with macroH2A enrichment are not expressed; however, macroH2A depletion did not cause their up-regulation. Somewhat surprisingly, activating roles have also been reported for macroH2A, such as serum starvation-induced genes (Fig. 3; Gamble et al. 2010). Currently, the mechanism for this dual role of macroH2A remains unclear. It is possible that some of these discrepancies can be explained by macroH2A.1.1 inhibition of PARP-1 (Ouararhni et al. 2006) or poly(ADP-ribose) modification, both of which are known to be involved in transcriptional regulation.

\section{H3.3: a dynamic memory}

The function of H3.3 in transcription remains somewhat unclear. Studies in Tetrahymena showed that H3.3 is not essential for transcription or viability (Cui et al. 2006). In adult Drosophila males, the loss of both H3.3 genes results in partial lethality and mostly affects highly expressed genes, with more genes up-regulated than down-regulated overall (Sakai et al. 2009). However, constitutive expression of $\mathrm{H} 3$ largely rescued these effects, suggesting that transcription differences were a consequence of nucleosome depletion and not specifically H3.3. In H3.3-depleted or HIRA ${ }^{-1-}$ ESCs, a minor fraction of genes showed differences in transcript levels, some of which are developmentally regulated; however, there is a much larger effect when H3.3 is depleted in partially differentiated mouse embryonic fibroblasts (Goldman et al. 2010; Banaszynski et al. 2013).

Recent evidence suggests that there are unique roles for H3.3 and its interacting partners in transcription. For example, H3.3 is important for early gene activation in cell lines and during myogenic differentiation (Placek et al. 2009; Tamura et al. 2009; Yang et al. 2011). In Xenopus, HIRA-mediated deposition of H3.3 is required for the transcriptional memory of active genes after somatic cell transfer into enucleated eggs ( $\mathrm{Ng}$ and Gurdon 2008; Jullien et al. 2012). H3.3 has also been shown to prime genes for later activation after genotoxic stress (Adam et al. 2013). Although the molecular basis for transcriptional memory is unknown, it is intriguing that $\mathrm{H} 3.3 / \mathrm{H} 4$ tetramers at human enhancer elements split during replication (Huang et al. 2013). H3.3 deposition might facilitate transcription factor binding by keeping these regions of the genome accessible (Fig. 3). Experiments in ESCs also support this general role, where H3.3 knockdown compromised PRC2 binding as well as H3K27me3 at "bivalent promoters" (Banaszynski et al. 2013). A similar role has been ascribed to H2A.Z, and it is thus conceivable that there is some cross-talk or that increased H2A.Z dynamics influence H3.3 deposition and dynamics.

\section{Perspective}

Over the past few years, there has been a growing appreciation for histone variants in transcriptional regulation. From the evidence described in this review, chromatinmediated gene regulation acts primarily through modulation of nucleosome dynamics and access to the underlying DNA. Although variants are structurally distinct from their canonical counterparts, replacement of a canonical histone with a variant is not the only way to alter the stability and dynamics of nucleosomes. An emerging theme is that variants function as an ensemble, coordinately modifying nucleosome properties and interacting with an expanding catalog of other factors within the nucleus. Differences in physical properties of variants and interactions with trans-acting factors result in the dynamic punctuation of chromatin that profoundly influences accessibility of the genome and, ultimately, transcriptional regulation. This is perhaps most evident during development in metazoans, which involves global changes in chromatin organization and transcriptional programs. A major area of interest for the future will be the characterization of metazoan deposition complexes, where we anticipate many context-dependent roles and the emergence of many additional players. 


\section{References}

Abbott DW, Chadwick BP, Thambirajah AA, Ausio J. 2005. Beyond the Xi: macroH2A chromatin distribution and posttranslational modification in an avian system. I Biol Chem 280: $16437-16445$.

Adam M, Robert F, Larochelle M, Gaudreau L. 2001. H2A.Z is required for global chromatin integrity and for recruitment of RNA polymerase II under specific conditions. Mol Cell Biol 21: 6270-6279.

Adam S, Polo SE, Almouzni G. 2013. Transcription recovery after DNA damage requires chromatin priming by the $\mathrm{H} 3.3$ histone chaperone HIRA. Cell 155: 94-106.

Agelopoulos M, Thanos D. 2006. Epigenetic determination of a cell-specific gene expression program by ATF- 2 and the histone variant macroH2A. EMBO J 25: 4843-4853.

Ahmad K, Henikoff S. 2002. The histone variant H3.3 marks active chromatin by replication-independent nucleosome assembly. Mol Cell 9: 1191-1200.

Albert I, Mavrich TN, Tomsho LP, Qi J, Zanton SJ, Schuster SC, Pugh BF. 2007. Translational and rotational settings of H2A.Z nucleosomes across the Saccharomyces cerevisiae genome. Nature 446: 572-576.

Allis CD, Glover CV, Bowen JK, Gorovsky MA. 1980. Histone variants specific to the transcriptionally active, amitotically dividing macronucleus of the unicellular eucaryote, Tetrahymena thermophila. Cell 20: 609-617.

Angelov D, Molla A, Perche PY, Hans F, Cote J, Khochbin S, Bouvet P, Dimitrov S. 2003. The histone variant macroH2A interferes with transcription factor binding and SWI/SNF nucleosome remodeling. Mol Cell 11: 1033-1041.

Banaszynski LA, Wen D, Dewell S, Whitcomb SJ, Lin M, Diaz N, Elsasser SJ, Chapgier A, Goldberg AD, Canaani E, et al. 2013. Hira-dependent histone H3.3 deposition facilitates PRC2 recruitment at developmental loci in ES cells. Cell 155: $107-120$.

Bao Y, Konesky K, Park YJ, Rosu S, Dyer PN, Rangasamy D, Tremethick DJ, Laybourn PJ, Luger K. 2004. Nucleosomes containing the histone variant H2A.Bbd organize only 118 base pairs of DNA. EMBO / 23: 3314-3324.

Billon P, Cote J. 2012. Precise deposition of histone H2A.Z in chromatin for genome expression and maintenance. Biochim Biophys Acta 1819: 290-302.

Biswas D, Dutta-Biswas R, Mitra D, Shibata Y, Strahl BD, Formosa T, Stillman DJ. 2006. Opposing roles for Set2 and yFACT in regulating TBP binding at promoters. EMBO J 25: 4479-4489.

Bonisch C, Hake SB. 2012. Histone H2A variants in nucleosomes and chromatin: more or less stable? Nucleic Acids Res 40: 10719-10741.

Bruce K, Myers FA, Mantouvalou E, Lefevre P, Greaves I, Bonifer C, Tremethick DJ, Thorne AW, Crane-Robinson C. 2005. The replacement histone H2A.Z in a hyperacetylated form is a feature of active genes in the chicken. Nucleic Acids Res 33: 5633-5639.

Buschbeck M, Uribesalgo I, Wibowo I, Rue P, Martin D, Gutierrez A, Morey L, Guigo R, Lopez-Schier H, Di Croce L. 2009. The histone variant macroH2A is an epigenetic regulator of key developmental genes. Nat Struct Mol Biol 16: 1074-1079.

Chadwick BP, Valley CM, Willard HF. 2001. Histone variant macroH2A contains two distinct macrochromatin domains capable of directing macroH2A to the inactive X chromosome. Nucleic Acids Res 29: 2699-2705.

Chakravarthy S, Luger K. 2006. The histone variant macro-H2A preferentially forms 'hybrid nucleosomes'. J Biol Chem 281: 25522-25531.
Chakravarthy S, Bao Y, Roberts VA, Tremethick D, Luger K. 2004. Structural characterization of histone H2A variants. Cold Spring Harb Symp Quant Biol 69: 227-234.

Chakravarthy S, Gundimella SK, Caron C, Perche PY, Pehrson JR, Khochbin S, Luger K. 2005. Structural characterization of the histone variant macroH2A. Mol Cell Biol 25: 7616-7624.

Chang EY, Ferreira H, Somers J, Nusinow DA, Owen-Hughes T, Narlikar GJ. 2008. MacroH2A allows ATP-dependent chromatin remodeling by SWI/SNF and ACF complexes but specifically reduces recruitment of SWI/SNF. Biochemistry 47: 13726-13732.

Changolkar LN, Costanzi C, Leu NA, Chen D, McLaughlin KJ, Pehrson JR. 2007. Developmental changes in histone macroH2A1-mediated gene regulation. Mol Cell Biol 27: $2758-2764$.

Changolkar LN, Singh G, Pehrson JR. 2008. macroH2A1-dependent silencing of endogenous murine leukemia viruses. Mol Cell Biol 28: 2059-2065.

Chen P, Zhao J, Wang Y, Wang M, Long H, Liang D, Huang L, Wen Z, Li W, Li X, et al. 2013. H3.3 actively marks enhancers and primes gene transcription via opening higher-ordered chromatin. Genes Dev 27: 2109-2124.

Chen Y, Chen Q, McEachin R, Cavalcoli J, Yu X. 2014. H2A.B facilitates transcription elongation at methylated $\mathrm{CpG}$ loci. Genome Res doi: 10.1101/gr.156877.113.

Coleman-Derr D, Zilberman D. 2012. Deposition of histone variant H2A.Z within gene bodies regulates responsive genes. PLoS Genet 8: e1002988.

Conerly ML, Teves SS, Diolaiti D, Ulrich M, Eisenman RN, Henikoff S. 2010. Changes in H2A.Z occupancy and DNA methylation during B-cell lymphomagenesis. Genome Res 20: $1383-1390$.

Core LJ, Waterfall JJ, Lis JT. 2008. Nascent RNA sequencing reveals widespread pausing and divergent initiation at human promoters. Science 322: 1845-1848.

Costanzi C, Pehrson JR. 1998. Histone macroH2A1 is concentrated in the inactive $\mathrm{X}$ chromosome of female mammals. Nature 393: 599-601.

Creyghton MP, Markoulaki S, Levine SS, Hanna J, Lodato MA, Sha K, Young RA, Jaenisch R, Boyer LA. 2008. H2AZ is enriched at polycomb complex target genes in ES cells and is necessary for lineage commitment. Cell 135: 649-661.

Csankovszki G, Panning B, Bates B, Pehrson JR, Jaenisch R. 1999. Conditional deletion of Xist disrupts histone macroH2A localization but not maintenance of X inactivation. Nat Genet 22: 323-324.

Cui B, Liu Y, Gorovsky MA. 2006. Deposition and function of histone $\mathrm{H} 3$ variants in Tetrahymena thermophila. Mol Cell Biol 26: 7719-7730.

Deal RB, Henikoff JG, Henikoff S. 2010. Genome-wide kinetics of nucleosome turnover determined by metabolic labeling of histones. Science 328: 1161-1164.

Dhillon N, Kamakaka RT. 2000. A histone variant, Htzlp, and a Sirlp-like protein, Esc2p, mediate silencing at HMR. Mol Cell 6: 769-780.

Dion MF, Kaplan T, Kim M, Buratowski S, Friedman N, Rando OJ. 2007. Dynamics of replication-independent histone turnover in budding yeast. Science 315: 1405-1408.

Doyen CM, An W, Angelov D, Bondarenko V, Mietton F, Studitsky VM, Hamiche A, Roeder RG, Bouvet P, Dimitrov S. 2006a. Mechanism of polymerase II transcription repression by the histone variant macroH2A. Mol Cell Biol 26: 1156-1164.

Doyen CM, Montel F, Gautier T, Menoni H, Claudet C, Delacour-Larose M, Angelov D, Hamiche A, Bednar J, Faivre-Moskalenko C, et al. 2006b. Dissection of the unusual 
structural and functional properties of the variant H2A.Bbd nucleosome. EMBO J 25: 4234-4244.

Drane P, Ouararhni K, Depaux A, Shuaib M, Hamiche A. 2010. The death-associated protein DAXX is a novel histone chaperone involved in the replication-independent deposition of H3.3. Genes Dev 24: 1253-1265.

Elsasser SJ, Huang H, Lewis PW, Chin JW, Allis CD, Patel DJ. 2012. DAXX envelops a histone H3.3-H4 dimer for H3.3specific recognition. Nature 491: 560-565.

Filipescu D, Szenker E, Almouzni G. 2013. Developmental roles of histone H3 variants and their chaperones. Trends Genet 29: 630-640.

Gallant-Behm CL, Ramsey MR, Bensard CL, Nojek I, Tran J, Liu M, Ellisen LW, Espinosa JM. 2012. $\Delta$ Np63 $\alpha$ represses antiproliferative genes via H2A.Z deposition. Genes Dev 26: 2325-2336.

Gamble MJ, Frizzell KM, Yang C, Krishnakumar R, Kraus WL. 2010. The histone variant macroH2A1 marks repressed autosomal chromatin, but protects a subset of its target genes from silencing. Genes Dev 24: 21-32.

Gautier T, Abbott DW, Molla A, Verdel A, Ausio J, Dimitrov S. 2004. Histone variant H2ABbd confers lower stability to the nucleosome. EMBO Rep 5: 715-720.

Gevry N, Chan HM, Laflamme L, Livingston DM, Gaudreau L. 2007. p21 transcription is regulated by differential localization of histone H2A.Z. Genes Dev 21: 1869-1881.

Goldberg AD, Banaszynski LA, Noh KM, Lewis PW, Elsaesser SJ, Stadler S, Dewell S, Law M, Guo X, Li X, et al. 2010. Distinct factors control histone variant $\mathrm{H} 3.3$ localization at specific genomic regions. Cell 140: 678-691.

Goldman JA, Garlick JD, Kingston RE. 2010. Chromatin remodeling by imitation switch (ISWI) class ATP-dependent remodelers is stimulated by histone variant H2A.Z. I Biol Chem 285: 4645-4651.

Guillemette B, Bataille AR, Gevry N, Adam M, Blanchette M, Robert F, Gaudreau L. 2005. Variant histone H2A.Z is globally localized to the promoters of inactive yeast genes and regulates nucleosome positioning. PLoS Biol 3: e384.

Halley JE, Kaplan T, Wang AY, Kobor MS, Rine J. 2010. Roles for H2A.Z and its acetylation in GAL1 transcription and gene induction, but not GAL1-transcriptional memory. PLoS Biol 8: e1000401

Han W, Li X, Fu X. 2011. The macro domain protein family: structure, functions, and their potential therapeutic implications. Mutat Res 727: 86-103.

Hardy S, Jacques PE, Gevry N, Forest A, Fortin ME, Laflamme L, Gaudreau L, Robert F. 2009. The euchromatic and heterochromatic landscapes are shaped by antagonizing effects of transcription on H2A.Z deposition. PLoS Genet 5: e1000687.

Hartley PD, Madhani HD. 2009. Mechanisms that specify promoter nucleosome location and identity. Cell 137: 445458

Hu G, Cui K, Northrup D, Liu C, Wang C, Tang Q, Ge K, Levens D, Crane-Robinson C, Zhao K. 2013. H2A.Z facilitates access of active and repressive complexes to chromatin in embryonic stem cell self-renewal and differentiation. Cell Stem Cell 12: 180-192.

Huang C, Zhang Z, Xu M, Li Y, Li Z, Ma Y, Cai T, Zhu B. 2013. H3.3-H4 tetramer splitting events feature cell-type specific enhancers. PLoS Genet 9: e1003558.

Illingworth RS, Botting CH, Grimes GR, Bickmore WA, Eskeland R. 2012. PRC1 and PRC2 are not required for targeting of H2A.Z to developmental genes in embryonic stem cells. PLOS ONE 7: e34848.

Ishibashi T, Li A, Eirin-Lopez JM, Zhao M, Missiaen K, Abbott DW, Meistrich M, Hendzel MJ, Ausio J. 2010. H2A.Bbd: an
X-chromosome-encoded histone involved in mammalian spermiogenesis. Nucleic Acids Res 38: 1780-1789.

Jamai A, Imoberdorf RM, Strubin M. 2007. Continuous histone $\mathrm{H} 2 \mathrm{~B}$ and transcription-dependent histone $\mathrm{H} 3$ exchange in yeast cells outside of replication. Mol Cell 25: 345-355

Jin C, Felsenfeld G. 2007. Nucleosome stability mediated by histone variants H3.3 and H2A.Z. Genes Dev 21: 15191529.

Jin C, Zang C, Wei G, Cui K, Peng W, Zhao K, Felsenfeld G. 2009. H3.3/H2A.Z double variant-containing nucleosomes mark 'nucleosome-free regions' of active promoters and other regulatory regions. Nat Genet 41: 941-945.

Jullien J, Astrand C, Szenker E, Garrett N, Almouzni G, Gurdon JB. 2012. HIRA dependent H3.3 deposition is required for transcriptional reprogramming following nuclear transfer to Xenopus oocytes. Epigenetics Chromatin 5: 17.

Kelly TK, Miranda TB, Liang G, Berman BP, Lin JC, Tanay A, Jones PA. 2010. H2A.Z maintenance during mitosis reveals nucleosome shifting on mitotically silenced genes. Mol Cell 39: 901-911.

Kimura H, Cook PR. 2001. Kinetics of core histones in living human cells: little exchange of $\mathrm{H} 3$ and $\mathrm{H} 4$ and some rapid exchange of H2B. J Cell Biol 153: 1341-1353.

Kumar SV, Wigge PA. 2010. H2A.Z-containing nucleosomes mediate the thermosensory response in Arabidopsis. Cell 140: 136-147.

Li B, Pattenden SG, Lee D, Gutierrez J, Chen J, Seidel C, Gerton J, Workman JL. 2005a. Preferential occupancy of histone variant $\mathrm{H} 2 \mathrm{AZ}$ at inactive promoters influences local histone modifications and chromatin remodeling. Proc Natl Acad Sci 102: 18385-18390.

Li G, Levitus M, Bustamante C, Widom J. 2005b. Rapid spontaneous accessibility of nucleosomal DNA. Nat Struct Mol Biol 12: $46-53$

Li B, Carey M, Workman JL. 2007. The role of chromatin during transcription. Cell 128: 707-719.

Li Z, Gadue P, Chen K, Jiao Y, Tuteja G, Schug J, Li W, Kaestner KH. 2012. Foxa2 and H2A.Z mediate nucleosome depletion during embryonic stem cell differentiation. Cell 151: 16081616.

Luger K, Mader AW, Richmond RK, Sargent DF, Richmond TJ. 1997. Crystal structure of the nucleosome core particle at 2.8 A resolution. Nature 389: 251-260.

Luk E, Vu ND, Patteson K, Mizuguchi G, Wu WH, Ranjan A, Backus J, Sen S, Lewis M, Bai Y, et al. 2007. Chz1, a nuclear chaperone for histone H2AZ. Mol Cell 25: 357-368.

Luk E, Ranjan A, Fitzgerald PC, Mizuguchi G, Huang Y, Wei D, Wu C. 2010. Stepwise histone replacement by SWR1 requires dual activation with histone H2A.Z and canonical nucleosome. Cell 143: 725-736.

Malik HS, Henikoff S. 2003. Phylogenomics of the nucleosome. Nat Struct Biol 10: 882-891.

Marques M, Laflamme L, Gervais AL, Gaudreau L. 2010. Reconciling the positive and negative roles of histone H2A.Z in gene transcription. Epigenetics 5: 267-272.

Mavrich TN, Jiang C, Ioshikhes IP, Li X, Venters BJ, Zanton SJ, Tomsho LP, Qi J, Glaser RL, Schuster SC, et al. 2008. Nucleosome organization in the Drosophila genome. Nature 453: 358-362.

McKittrick E, Gafken PR, Ahmad K, Henikoff S. 2004. Histone H3.3 is enriched in covalent modifications associated with active chromatin. Proc Natl Acad Sci 101: 1525-1530.

Meneghini MD, Wu M, Madhani HD. 2003. Conserved histone variant $\mathrm{H} 2 \mathrm{~A} . \mathrm{Z}$ protects euchromatin from the ectopic spread of silent heterochromatin. Cell 112: 725-736. 
Mito Y, Henikoff JG, Henikoff S. 2007. Histone replacement marks the boundaries of cis-regulatory domains. Science 315: 1408-1411.

Mizuguchi G, Shen X, Landry J, Wu WH, Sen S, Wu C. 2004. ATP-driven exchange of histone $\mathrm{H} 2 \mathrm{AZ}$ variant catalyzed by SWR1 chromatin remodeling complex. Science 303: 343-348.

Morillo-Huesca M, Clemente-Ruiz M, Andujar E, Prado F. 2010. The SWR1 histone replacement complex causes genetic instability and genome-wide transcription misregulation in the absence of H2A.Z. PLOS ONE 5: e12143.

Nekrasov M, Amrichova J, Parker BJ, Soboleva TA, Jack C, Williams R, Huttley GA, Tremethick DJ. 2012. Histone H2A.Z inheritance during the cell cycle and its impact on promoter organization and dynamics. Nat Struct Mol Biol 19: $1076-1083$

Ng RK, Gurdon JB. 2008. Epigenetic inheritance of cell differentiation status. Cell Cycle 7: 1173-1177.

Nusinow DA, Sharp JA, Morris A, Salas S, Plath K, Panning B. 2007. The histone domain of macroH2A1 contains several dispersed elements that are each sufficient to direct enrichment on the inactive X chromosome. J Mol Biol 371: 11-18.

Obri A, Ouararhni K, Papin C, Diebold ML, Padmanabhan K, Marek M, Stoll I, Roy L, Reilly PT, Mak TW, et al. 2014. ANP32E is a histone chaperone that removes H2A.Z from chromatin. Nature 505: 648-653.

Okuwaki M, Kato K, Shimahara H, Tate S, Nagata K. 2005. Assembly and disassembly of nucleosome core particles containing histone variants by human nucleosome assembly protein I. Mol Cell Biol 25: 10639-10651.

Ouararhni K, Hadj-Slimane R, Ait-Si-Ali S, Robin P, Mietton F, Harel-Bellan A, Dimitrov S, Hamiche A. 2006. The histone variant $\mathrm{mH} 2 \mathrm{~A} 1.1$ interferes with transcription by downregulating PARP-1 enzymatic activity. Genes Dev 20: 3324-3336.

Papamichos-Chronakis M, Watanabe S, Rando OJ, Peterson CL. 2011. Global regulation of H2A.Z localization by the INO80 chromatin-remodeling enzyme is essential for genome integrity. Cell 144: 200-213.

Pchelintsev NA, McBryan T, Rai TS, van Tuyn J, Ray-Gallet D, Almouzni G, Adams PD. 2013. Placing the HIRA histone chaperone complex in the chromatin landscape. Cell Rep 3: 1012-1019.

Pina B, Suau P. 1987. Changes in histones H2A and H3 variant composition in differentiating and mature rat brain cortical neurons. Dev Biol 123: 51-58.

Placek BJ, Huang J, Kent JR, Dorsey J, Rice L, Fraser NW, Berger SL. 2009. The histone variant H3.3 regulates gene expression during lytic infection with herpes simplex virus type 1. I Virol 83: $1416-1421$.

Ranjan A, Mizuguchi G, FitzGerald PC, Wei D, Wang F, Huang Y, Luk E, Woodcock CL, Wu C. 2013. Nucleosome-free region dominates histone acetylation in targeting SWR1 to promoters for H2A.Z replacement. Cell 154: 1232-1245.

Ratnakumar K, Duarte LF, LeRoy G, Hasson D, Smeets D, Vardabasso C, Bonisch C, Zeng T, Xiang B, Zhang DY, et al. 2012. ATRX-mediated chromatin association of histone variant macroH2A1 regulates $\alpha$-globin expression. Genes Dev 26: 433-438.

Ray-Gallet D, Woolfe A, Vassias I, Pellentz C, Lacoste N, Puri A, Schultz DC, Pchelintsev NA, Adams PD, Jansen LE, et al. 2011. Dynamics of histone H3 deposition in vivo reveal a nucleosome gap-filling mechanism for H3.3 to maintain chromatin integrity. Mol Cell 44: 928-941.

Rhee HS, Pugh BF. 2012. Genome-wide structure and organization of eukaryotic pre-initiation complexes. Nature 483: 295-301.
Sakai A, Schwartz BE, Goldstein S, Ahmad K. 2009. Transcriptional and developmental functions of the H3.3 histone variant in Drosophila. Curr Biol 19: 1816-1820.

Santisteban MS, Kalashnikova T, Smith MM. 2000. Histone H2A.Z regulates transcription and is partially redundant with nucleosome remodeling complexes. Cell 103: 411-422.

Santisteban MS, Hang M, Smith MM. 2011. Histone variant H2A.Z and RNA polymerase II transcription elongation. Mol Cell Biol 31: 1848-1860.

Sarcinella E, Zuzarte PC, Lau PN, Draker R, Cheung P. 2007. Monoubiquitylation of H2A.Z distinguishes its association with euchromatin or facultative heterochromatin. Mol Cell Biol 27: 6457-6468.

Sawatsubashi S, Murata T, Lim J, Fujiki R, Ito S, Suzuki E, Tanabe M, Zhao Y, Kimura S, Fujiyama S, et al. 2010. A histone chaperone, DEK, transcriptionally coactivates a nuclear receptor. Genes Dev 24: 159-170.

Schneiderman JI, Orsi GA, Hughes KT, Loppin B, Ahmad K. 2012. Nucleosome-depleted chromatin gaps recruit assembly factors for the H3.3 histone variant. Proc Natl Acad Sci 109: 19721-19726.

Sheinin MY, Li M, Soltani M, Luger K, Wang MD. 2013. Torque modulates nucleosome stability and facilitates $\mathrm{H} 2 \mathrm{~A} / \mathrm{H} 2 \mathrm{~B}$ dimer loss. Nat Commun 4: 2579.

Soboleva TA, Nekrasov M, Pahwa A, Williams R, Huttley GA, Tremethick DJ. 2012. A unique H2A histone variant occupies the transcriptional start site of active genes. Nat Struct Mol Biol 19: 25-30.

Suto RK, Clarkson MJ, Tremethick DJ, Luger K. 2000. Crystal structure of a nucleosome core particle containing the variant histone H2A.Z. Nat Struct Biol 7: 1121-1124.

Tagami H, Ray-Gallet D, Almouzni G, Nakatani Y. 2004. Histone H3.1 and H3.3 complexes mediate nucleosome assembly pathways dependent or independent of DNA synthesis. Cell 116: 51-61.

Talbert PB, Henikoff S. 2010. Histone variants-ancient wrap artists of the epigenome. Nat Rev Mol Cell Biol 11: 264-275.

Tamura T, Smith M, Kanno T, Dasenbrock H, Nishiyama A, Ozato K. 2009. Inducible deposition of the histone variant H3.3 in interferon-stimulated genes. J Biol Chem 284: 12217-12225.

Thakar A, Gupta P, Ishibashi T, Finn R, Silva-Moreno B, Uchiyama S, Fukui K, Tomschik M, Ausio J, Zlatanova J. 2009. H2A.Z and H3.3 histone variants affect nucleosome structure: biochemical and biophysical studies. Biochemistry 48: 10852-10857.

Tochio N, Umehara T, Munemasa Y, Suzuki T, Sato S, Tsuda K, Koshiba S, Kigawa T, Nagai R, Yokoyama S. 2010. Solution structure of histone chaperone ANP32B: interaction with core histones H3-H4 through its acidic concave domain. J Mol Biol 401: 97-114.

Tolstorukov MY, Goldman JA, Gilbert C, Ogryzko V, Kingston RE, Park PJ. 2012. Histone variant H2A.Bbd is associated with active transcription and mRNA processing in human cells. Mol Cell 47: 596-607.

Valdes-Mora F, Song JZ, Statham AL, Strbenac D, Robinson MD, Nair SS, Patterson KI, Tremethick DJ, Stirzaker C, Clark SJ. 2012. Acetylation of H2A.Z is a key epigenetic modification associated with gene deregulation and epigenetic remodeling in cancer. Genome Res 22: 307-321.

Viens A, Mechold U, Brouillard F, Gilbert C, Leclerc P, Ogryzko V. 2006. Analysis of human histone $\mathrm{H} 2 \mathrm{AZ}$ deposition in vivo argues against its direct role in epigenetic templating mechanisms. Mol Cell Biol 26: 5325-5335.

Watanabe S, Radman-Livaja M, Rando OJ, Peterson CL. 2013. A histone acetylation switch regulates H2A.Z deposition by the SWR-C remodeling enzyme. Science 340: 195-199. 
Weber CM, Henikoff JG, Henikoff S. 2010. H2A.Z nucleosomes enriched over active genes are homotypic. Nat Struct Mol Biol 17: 1500-1507.

Weber CM, Ramachandran S, Henikoff S. 2014. Nucleosomes are context-specific, H2A.Z modulated barriers to RNA polymerase. Mol Cell(in press).

Whitehouse I, Rando OJ, Delrow J, Tsukiyama T. 2007. Chromatin remodelling at promoters suppresses antisense transcription. Nature 450: 1031-1035.

Xie W, Song C, Young NL, Sperling AS, Xu F, Sridharan R, Conway AE, Garcia BA, Plath K, Clark AT, et al. 2009. Histone h3 lysine 56 acetylation is linked to the core transcriptional network in human embryonic stem cells. Mol Cell 33: 417-427.

Xu F, Zhang K, Grunstein M. 2005. Acetylation in histone H3 globular domain regulates gene expression in yeast. Cell 121: 375-385.

Xu Z, Wei W, Gagneur J, Perocchi F, Clauder-Munster S, Camblong J, Guffanti E, Stutz F, Huber W, Steinmetz LM. 2009. Bidirectional promoters generate pervasive transcription in yeast. Nature 457: 1033-1037.

Yang JH, Song Y, Seol JH, Park JY, Yang YJ, Han JW, Youn HD, Cho EJ. 2011. Myogenic transcriptional activation of MyoD mediated by replication-independent histone deposition. Proc Natl Acad Sci 108: 85-90.

Yen K, Vinayachandran V, Pugh BF. 2013. SWR-C and INO80 chromatin remodelers recognize nucleosome-free regions near +1 nucleosomes. Cell 154: 1246-1256.

Zemach A, McDaniel IE, Silva P, Zilberman D. 2010. Genomewide evolutionary analysis of eukaryotic DNA methylation. Science 328: 916-919.

Zhang R, Poustovoitov MV, Ye X, Santos HA, Chen W, Daganzo SM, Erzberger JP, Serebriiskii IG, Canutescu AA, Dunbrack RL, et al. 2005. Formation of MacroH2A-containing senescenceassociated heterochromatin foci and senescence driven by ASF1a and HIRA. Dev Cell 8: 19-30.

Zhou J, Fan JY, Rangasamy D, Tremethick DJ. 2007. The nucleosome surface regulates chromatin compaction and couples it with transcriptional repression. Nat Struct Mol Biol 14: 1070-1076.

Zilberman D, Coleman-Derr D, Ballinger T, Henikoff S. 2008. Histone H2A.Z and DNA methylation are mutually antagonistic chromatin marks. Nature 456: 125-129.

Zlatanova J, Thakar A. 2008. H2A.Z: view from the top. Structure 16: 166-179. 


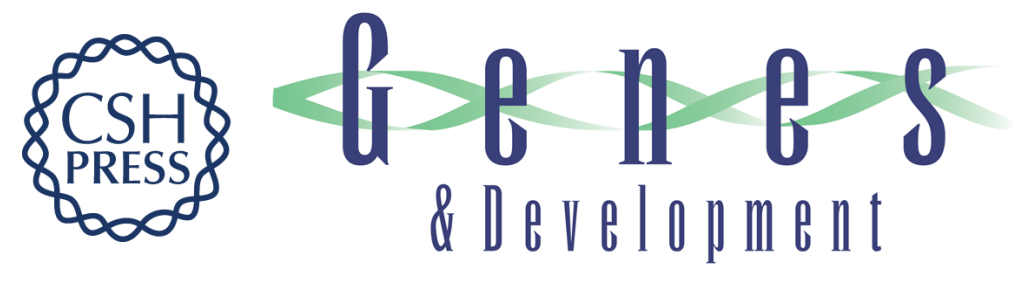

\section{Histone variants: dynamic punctuation in transcription}

Christopher M. Weber and Steven Henikoff

Genes Dev. 2014, 28:

Access the most recent version at doi:10.1101/gad.238873.114

References This article cites 119 articles, 43 of which can be accessed free at: http://genesdev.cshlp.org/content/28/7/672.full.html\#ref-list-1

Creative This article, published in Genes \& Development, is available under a Creative Commons

Commons License (Attribution 4.0 International), as described at

License http://creativecommons.org/licenses/by/4.0.

Email Alerting Receive free email alerts when new articles cite this article - sign up in the box at the top Service right corner of the article or click here.

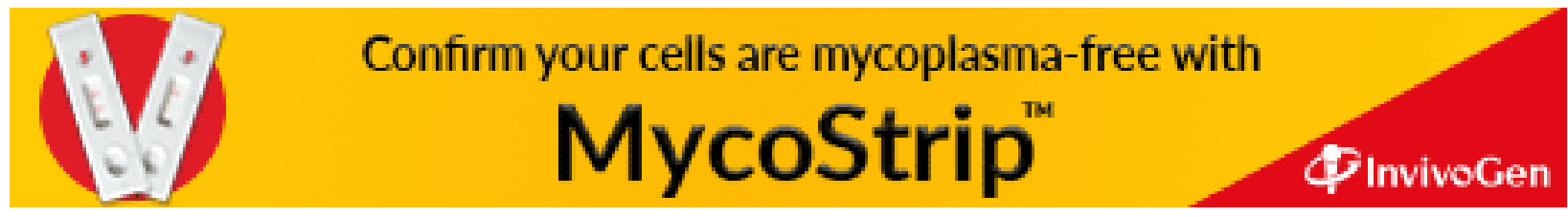

(C) 2014 Weber and Henikoff; Published by Cold Spring Harbor Laboratory Press 